\title{
Trajetória dos adolescentes usuários de crack até o Centro de Atenção Psicossocial Álcool e Drogas
}

The trajectory of adolescent crack users until the Psychosocial Care Center for Alcohol and Drugs

Trayectoria de los adolescentes usuarios de crack hasta el Centro de Atención Psicosocial Alcohol y

Drogas

\author{
Juliane Portella Ribeiro', Giovana Calcagno Gomes ${ }^{\mathrm{II}}$, Adriane Domingues Eslabão ${ }^{\mathrm{III}}$ \\ Naiana Alves Oliveira ${ }^{\mathrm{IV}}$
}

\begin{abstract}
Resumo: Objetivo: analisar a trajetória percorrida pelos adolescentes usuários de crack até o início do tratamento no Centro de Atenção Psicossocial Álcool e Drogas (CAPS AD). Método: trata-se de um estudo exploratório descritivo com abordagem qualitativa. Participaram usuários, familiares e profissionais atuantes no CAPS AD. Os dados foram coletados por meio de entrevistas semiestruturadas, organizados e tratados pelo software Nvivo $11 \mathrm{e}$, posteriormente, submetidos a Análise Temática. Resultado: a partir da análise dos dados emergiram as seguintes categorias: a forma como o adolescente usuário de crack chega ao CAPS AD; Motivos que levam o adolescente usuário de crack para tratamento no CAPS AD e Serviços utilizados pelos adolescentes usuários de crack até chegar ao CAPS AD. Considerações finais: mostra-se importante o investimento nos serviços e ações desenvolvidas pela Rede de Atenção Piscossocial (RAPS), dando-lhes maior visibilidade e acesso aos jovens.

Descritores: Adolescente; Cocaína Crack; Centros de Tratamento de Abuso de Substâncias; Reabilitação Psiquiátrica; Enfermagem
\end{abstract}

\begin{abstract}
Aim: to analyze the trajectory experienced by adolescent crack users until the beginning of their treatment in the Psychosocial Care Center for Alcohol and Drugs (CAPS AD). Method: This is a descriptive exploratory study, with a qualitative approach. Users, their relatives and workers from CAPS AD participated in the study. Data were collected through semi-structured interviews, organized and treated by Nvivo 11 software and,
\end{abstract}

\footnotetext{
${ }^{\text {I }}$ Enfermeira. Doutora em Enfermagem. Universidade Federal de Pelotas, Pelotas, RS, Brasil. E-mail: ju_ribeiro1985@hotmail.com Orcid: https://orcid.org/0000-0002-1882-6762

II Enfermeira. Doutora em Filosofia. Universidade Federal do Rio Grande, Rio Grande, RS, Brasil. E-mail: giovanacalcagno@furg.br Orcid: https://orcid.org/0000-0002-2464-1537

III Enfermeira. Mestre em Enfermagem. Universidade Federal do Rio Grande do Sul, Porto Alegre, RS, Brasil. E-mail: adrianeeslabao@hotmail.com Orcid: https://orcid.org/0000-0003-1262-6521

IV Enfermeira. Doutora em Ciências. Universidade Federal de Pelotas, Pelotas, RS, Brasil. E-mail: naivesoli@gmail.com Orcid: https://orcid.org/0000-0001-5116-9082
} 
subsequently, submitted to Thematic Analysis. Result: From data analysis, the following categories emerged: The way the adolescent crack user arrives at CAPS AD; Reasons that lead the adolescent crack user to seek treatment at CAPS AD and Services used by adolescent crack users until they arrive at CAPS AD. Final considerations: it is important to invest in services and actions developed by the Psychosocial Care Network (RAPS), giving them greater visibility and access to young people.

Descriptors: Adolescent; Crack Cocaine; Substance Abuse Treatment Centers; Psychiatric Rehabilitation; Nursing

Resumen: Objetivo: analizar la trayectoria de los adolescentes usuarios de crack hasta empezar el tratamiento en el Centro de Atención Psicosocial Alcohol y Drogas (CAPS AD). Método: se trata de un estudio exploratorio, descriptivo, de base cualitativo. Participaron del estudio usuarios, familiares y profesionales actuantes en el CAPS AD. Los datos fueron recolectados por medio de entrevistas semiestructuradas y ordenados y tratados por el software Nvivo $11 \mathrm{y}$, posteriormente, sometidos al Análisis Temático. Resultado: a partir del análisis de los datos surgieron las siguientes categorías: la forma que el adolescente, usuario de crack, llega al CAPS AD; los motivos que llevan el adolescente, usuario de crack, a tratarse en el CAPS AD; y los servicios utilizados por los adolescentes, usuarios de crack, hasta llegar al CAPS AD. Consideraciones finales: se reveló importante la inversión en los servicios y acciones desarrolladas por la Red de Atención Psicosocial (RAPS), dando a esos mayor visibilidad y acceso a los jóvenes.

Descriptores: Adolescentes; Cocaína; Crack; Centros de tratamiento de abuso de substancias; Rehabilitación psiquiátrica; Enfermería.

\section{Introdução}

O processo de Reforma Psiquiátrica é um movimento mundial de luta por melhores condições de cuidado em saúde mental. Tal movimento, a partir da década de 1960, começou a concretizar-se por meio de experiências que vão desde transformações no interior dos manicômios até as propostas de desospitalização, desinstitucionalização, fechamentos de manicômios e a criação de uma rede de cuidado em saúde mental no âmbito comunitário. ${ }^{1}$

No cenário brasileiro, o Ministério da Saúde promulgou a Portaria n.ํㅜ 3.088/2011, a qual estabelece a Rede de Atenção Psicossocial (RAPS) para pessoas com sofrimento ou transtorno mental e com necessidades decorrentes do uso de crack, álcool e outras drogas. A RAPS é composta por serviços e equipamentos variados, tais como: os Centros de Atenção Psicossocial 
3 | Ribeiro JP,Gomes GC, Eslabão AD, Oliveira NA

(CAPS); os Serviços Residenciais Terapêuticos (SRT); os Centros de Convivência e Cultura, as Unidade de Acolhimento (UAs), e os leitos de atenção integral (em Hospitais Gerais e no CAPS III) ${ }^{2}$

A RAPS regula e organiza as demandas e os fluxos assistenciais entre os serviços de Atenção Psicossocial, enfatizando que as pessoas com problemas relacionados as drogas sejam atendidas nos vários pontos de atenção da rede, de acordo com a gravidade de sua situação, ficando as internações indicadas apenas para os casos graves de intoxicações, abstinência ou dependência com risco de vida, em que o usuário não consegue resolução de sua situação no atendimento extra-hospitalar. ${ }^{3}$

A RAPS pressupõem a resposta adequada a necessidade do usuário, encontrando dentro da rede o serviço apropriado para tal. Ela se constitui na relação e na pactuação coletiva, a qual concretiza-se por meio do reconhecimento e a corresposabilização do que compete a cada área, serviço, equipe, profissional. Assim, potencializando a organização dos processos de trabalho e dos fluxos de acesso e cuidado aos usuários. ${ }^{3}$

No entanto, é notória a lacuna existente na RAPS quanto ao cuidado do adolescente usuário de drogas. Tal entendimento se sustenta no fato de que ainda é incipiente a instalação de serviços especializados a esta população, tanto que o Centro de Atenção Psicossocial Infantil (CAPSi) como o Centro de Atenção Psicossocial Álcool e Drogas (CAPS AD) atendem esse 
Trajetória dos adolescentes usuários de crack até o centro de atenção psicossocial álcool e drogas | 4

público, o que, consequentemente, favorece a fragmentação e a desresponsabilização pela assistência e pelo cuidado. ${ }^{4}$

Nesse sentido, estudo realizado no interior do estado do Rio Grande do Sul, com profissionais atuantes em um serviço de Atenção Psicossocial para usuários de substancias psicoativas, apontou que o serviço prestado é deficitário em relação à estrutura, recursos humanos e materiais. Além disto, denotam que o ambiente é pouco atrativo para os adolescentes, visto que este público tem características e necessidades singulares, além da fragmentação da rede assistencial, bem como a escassa comunicação entre os prestadores dos serviços de saúde. ${ }^{5}$

O cuidado voltado aos adolescentes usuários de drogas destaca-se como necessidade premente, haja vista a escassez de locais adequados e de profissionais capacitados para atender as suas demandas. ${ }^{6}$ Somado a isso, o preconceito e o estigma com quem usa drogas como o crack, é outro ponto importante, pois acarreta o distanciamento dos jovens dos serviços de atenção em saúde. ${ }^{6}$

Pesquisa com o objetivo de avaliar o estigma e o preconceito vividos pelos usuários de crack em seu contexto social indicou que a sociedade percebe o usuário de crack a partir de uma identidade estigmatizante de marginal, vagabundo e violento, gerando a ideia de pessoa não cidadã, sem direito a um lugar social, aquele que deve ser excluído. Por essa razão, a mesma 
5 | Ribeiro JP,Gomes GC, Eslabão AD, Oliveira NA

propõe o tensionamento do preconceito diante do uso de drogas sem moralizá-lo, fato que poderia resultar em maior acesso dos usuários às redes de cuidado. ${ }^{7}$

Frente ao contexto, estudar a RAPS mostra-se relevante no contexto atual, tendo em vista que a fragmentação entre os serviços da rede direcionados ao cuidado do adolescente usuário de drogas gera um círculo vicioso de sucessivas ações isoladas que acabam por colocar em descrença a própria potência da rede.

Buscando proporcionar a reflexão aos profissionais sobre suas práticas de saúde voltadas aos adolescentes e possibilitar a reestruturação em seu trabalho conforme as necessidades dessa população e da Rede de Atenção, o presente estudo tem por objetivo analisar a trajetória percorrida pelos adolescentes usuários de crack até o tratamento no Centro de Atenção Psicossocial Álcool e Drogas (CAPS AD).

\section{Método}

Trata-se de um estudo exploratório e descritivo com abordagem qualitativa, vinculado a um projeto de pesquisa amplo, intitulado “(Des)caminhos percorridos pelo adolescente usuário de crack na rede de atenção psicossocial: contribuição para a Enfermagem”, desenvolvido em um município de médio porte do interior do Rio Grande do Sul que integra o Programa "Crack, é possível vencer”. 
Trajetória dos adolescentes usuários de crack até o centro de atenção psicossocial álcool e drogas I 6

O CAPS AD foi eleito como local de coleta de dados, uma vez que possui especificidades distintas da pluralidade dos demais serviços que compõem a RAPS do município. A maioria dos serviços atua de forma pontual e delimitada por tempo específico, enquanto que o CAPS AD é um serviço de atenção integral e continuada às pessoas com necessidades em decorrência do uso de álcool, crack e outras drogas. Além disso, na realidade da RAPS do município em questão, os casos de adolescentes usuários de drogas direcionados e acompanhados pelo CAPS infantil é ínfimo.

Os participantes deste estudo compreendem 14 usuários, 11 familiares e 20 profissionais atuantes no CAPS AD, totalizando 45 participantes. A seleção dos participantes foi intencional, de acordo com os critérios de inclusão e objetivos da pesquisa.

Para os usuários, os critérios de inclusão foram: ser adolescentes usuários de crack atendido no CAPS AD e ter entre 10 e 20 anos incompletos. Em relação ao primeiro critério, foram considerados aqueles adolescentes cujos prontuários fizeram menção ao consumo de substância pelo menos uma vez na vida, sem distinção quanto ao tempo e frequência de uso. Foram excluídos do estudo os adolescentes que estavam hospitalizados ou em comunidades terapêuticas e impossibilitados de comparecer a entrevista.

Para os familiares, os critérios de inclusão foram: ter idade mínima de 18 anos e ser familiar do usuário e responsável pelo tratamento do adolescente no CAPS AD. Foram excluídos do estudo familiares cuidadores eventuais do usuário. 
Para os profissionais do CAPS AD, os critérios de inclusão foram: ser trabalhador de nível médio ou superior que compõem a equipe multiprofissional e possuir no mínimo seis meses de atuação no serviço. Foram excluídos profissionais em férias ou licença saúde no período de coleta dos dados.

Com vistas a garantir os princípios éticos relativos a pesquisas que envolvem seres humanos, os participantes foram incluídos no estudo, somente, após manifestarem sua concordância em participar do mesmo por meio da assinatura do Termo de Consentimento Livre e Esclarecido (TCLE) e do Termo de Assentimento Livre e Esclarecido, quando menor de dezoito anos. Além disso, o anonimato dos participantes foi preservado por meio do emprego da letra “A” para os adolescentes usuários do CAPS AD, "F” para os respectivos familiares e "P" para os profissionais do serviço, sucedidas do número da entrevista.

O número de participantes foi definido pela saturação dos dados, estabelecida quando, na avaliação do pesquisador, ocorre certa redundância ou repetição dos achados, não sendo considerado relevante persistir na inclusão de participantes. A coleta de dados ocorreu no primeiro semestre de 2017, por meio de entrevistas semiestruturadas realizadas por um único entrevistador, uma vez que essa abordagem permite que o mesmo se coloque o mais próximo possível da perspectiva do entrevistado. ${ }^{8}$

Com vistas à privacidade dos participantes, as entrevistas foram realizadas em uma sala do serviço, respeitando a disponibilidade e funcionamento do CAPS AD. Para preservar o conteúdo 
Trajetória dos adolescentes usuários de crack até o centro de atenção psicossocial álcool e drogas $\mid 8$

original e aumentar a acurácia dos dados obtidos, as entrevistas foram capturadas por um gravador de áudio e, posteriormente, transcritas na íntegra.

Para a organização e tratamento dos dados, empregou-se o software QSR Nvivo 11, programa que auxilia na análise de material qualitativo com ferramentas de codificação e armazenamento de textos. ${ }^{9}$ Posteriormente, o material foi analisado e categorizado conforme a Análise Temática. ${ }^{10}$ Utilizou-se a ferramenta da nuvem de palavras, criada a partir do software QSR Nvivo 11, para visualizar a representatividade e ocorrência das mesmas em uma dada categoria, uma vez que esta técnica emprega diferentes tamanhos e fontes de letras para enfatizar a frequência da palavra no texto analisado.

Os preceitos éticos da realização de pesquisa envolvendo seres humanos foram respeitados conforme a Resolução n 466, de 12 de dezembro de $2012 .{ }^{11}$ O projeto foi aprovado por Comitê de Ética em Pesquisa mediante o Certificado de Apresentação para Apreciação Ética (CAEE) no 60649016.9.0000.5324, Parecer nำ 4/2017.

\section{Resultados}

A partir análise dos dados, acerca da trajetória percorrida pelos adolescentes usuários de crack até o tratamento no CAPS AD, emergiram as seguintes categorias: a forma como o adolescente usuário de crack chega ao CAPS AD; Motivos que levam o adolescente usuário de 
9 | Ribeiro JP,Gomes GC, Eslabão AD, Oliveira NA

crack para tratamento no CAPS AD e Serviços utilizados pelos adolescentes usuários de crack até chegar ao CAPS AD.

\section{A forma como o adolescente usuário de crack chega ao CAPS AD}

Os participantes revelaram que o adolescente chega ao CAPS AD de diferentes formas, sendo as mais frequentes: por demanda espontânea, acompanhado pelos responsáveis ou por representantes de órgãos de proteção e justiça, encaminhados por demais serviços da rede de atenção à saúde (Figura 1).

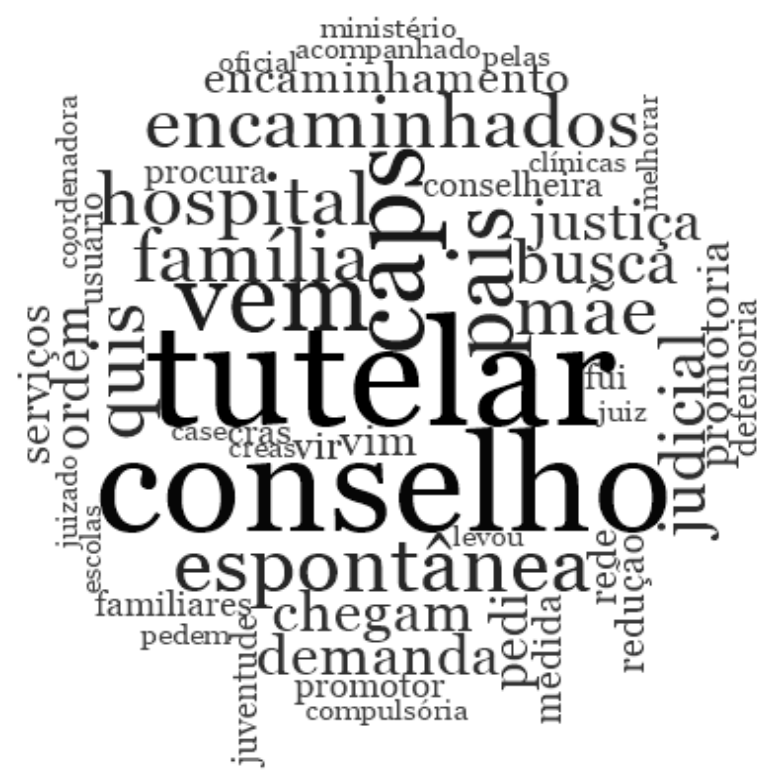

Figura 1 - Nuvem de palavras por frequência da codificação do sub-nó “A forma como o adolescente usuário de crack chega ao CAPS AD”, via QSR NVivo.

A demanda espontânea é explicitada por: eu pedi, quis, vontade própria, o adolescente mesmo busca, demanda espontânea, busca espontânea e procura espontânea. Os responsáveis que acompanham o adolescente são seus pais, familiares, pai ou mãe e coordenadora do abrigo.

Entre os representantes de órgãos de proteção e justiça destacam-se o conselho tutelar, conselheira tutelar, juiz, justiça, ordem judicial, medida judicial, oficial de justiça, promotor, promotoria, defensoria pública, ministério público, juizado da infância e juventude. 
Os meus pais e o Conselho Tutelar me trouxeram. De tanto me incomodar e dizer, aí eu vim. Também meus amigos morreram por causa da droga, aí eu fui e quis me ajudar também. (A4)

Eu quis, eu que pedi ajuda para a minha mãe. Aí minha mãe me ajudou e me trouxe para cá. (A6)

Na primeira vez eu cheguei no CAPS por intermédio do conselho tutelar. (F4)

Da outra vez ele fugiu e não quis. Agora ele está lá de novo. Ele foi, mas não porque ele quis, porque nós obrigamos. (F9)

São diversas as formas de encaminhamento dos adolescentes ao CAPS AD. A busca por atendimento junto ao CAPS AD se dá por busca espontânea ou encaminhamento da rede de serviços: redução de danos, CRAS, CREAS, CASE, juizado da infância e juventude, Conselho Tutelar, Hospital geral, Hospital psiquiátrico, entre outros. (P3)

Eles vem ou são encaminhados pelo CRAS, ou vêm encaminhados pelo conselho tutelar, ou após uma alta do hospital psiquiátrico ou eles vem com uma medida judicial para internar, ou até mesmo a familia que busca, ou o próprio adolescente que busca o CAPS como uma alternativa de tratamento para recuperação do uso da dependência química. (P11)

Os serviços da rede de atenção à saúde e da rede socioassistencial que realizaram encaminhamentos para o CAPS AD foram: CAPS i, unidades básicas, redução de danos, Centro de Referência de Assistência Social (CRAS), Centro de Referência Especializado de Assistência Social (CREAS), Centro de Atendimento a Saúde do Escolar (CASE), hospital geral, hospital psiquiátrico, clínicas, outros serviços, encaminhamento da rede.

\section{Motivos que levam o adolescente usuário de crack para tratamento no CAPS AD}

Os participantes revelaram que os motivos que levam os adolescentes para tratamento no CAPS AD incluem desde a necessidade do próprio adolescente em investir num projeto de vida melhor, pedidos da justiça e, principalmente, os problemas comportamentais (Figura 2). 


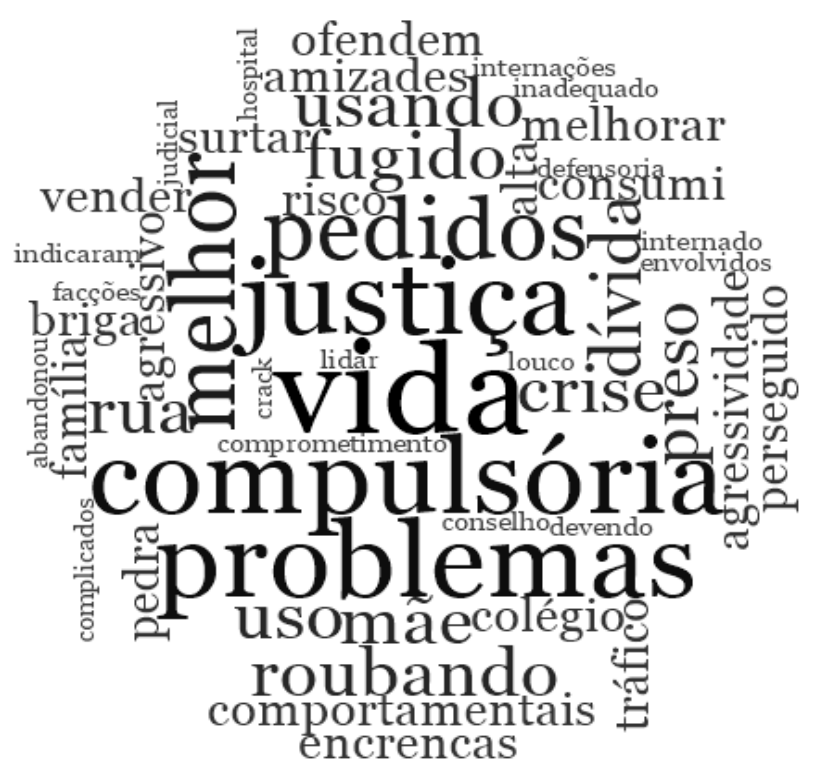

Figura 2 - Nuvem de palavras por frequência da codificação do sub-nó "Motivos que levam o adolescente usuário de crack para tratamento no CAPS AD”, via QSR NVivo.

A necessidade do próprio adolescente em investir num projeto de vida melhor foi revelado por meio das expressões: poder melhorar minha vida e ter uma vida melhor.

Eu mesma busquei [tratamento] por causa da minha filha. Eu que vim procurar o CAPS AD para poder melhorar minha vida e ajudar a minha filha que está no Abrigo, para ter uma vida melhor, eu e ela. (A2)

Os pedidos da justiça revelam-se pelas internações compulsórias:

Algumas vezes pela Promotoria, então o promotor aciona o Conselho Tutelar e ele vem por ordem judicial, inclusive internação compulsória. (P8)

A gente acompanha mais são os casos de adolescentes, os casos enviados por defensoria, pelos pais que pedem a internação compulsória. (P2)

Os problemas comportamentais são os principais motivos que levam os adolescentes para tratamento no CAPS AD, sendo citados: roubo, venda de artigos de casa, ser preso, uso de drogas, recaída e consumo de drogas, fugir de casa e ficar na rua, situações de crise ou surto, envolvimento com o tráfico, contração de dívidas, exposição à riscos, estar sendo perseguido, envolvimento em brigas e encrencas, problemas e agressividade. 
Já tinha me internado aqui. Eu ganhei alta e voltei para o abrigo, só que eu não podia ter ganhado alta porque eu ainda estava com a dúvida se eu iria conseguir e daí eu consumi de novo e voltei para cá. (A12)

Eu andei louco, aí eu fui preso e me mandaram para cá porque eu estava usando drogas. (A5)

Essa busca pelo tratamento foi devido ele ter fugido de casa. Ter fugido de casa, ter se misturado com gente que não deveria, ter usado drogas. (F8)

Eles vem para cá e situações de crise, quando eles vendem tudo ou quando eles estão demais. (P7)

Às vezes o adolescente vem para cá e ele está em risco, ele está devendo para traficante, está sendo perseguido. Vem bastante adolescentes assim, que tem problemas lá fora, com algumas facções que eles relatam que estão com alguma dívida, alguma briga. (P8)

\section{Serviços utilizados pelos adolescentes usuários de crack até chegar ao CAPS AD}

Os serviços utilizados com maior frequência pelos adolescentes usuários de crack até chegar ao CAPS AD revelam uma cultura de tratamento manicomial, em que raras exceções são acionados dispositivos públicos de proteção e atenção à saúde presentes na comunidade (Figura $3)$.

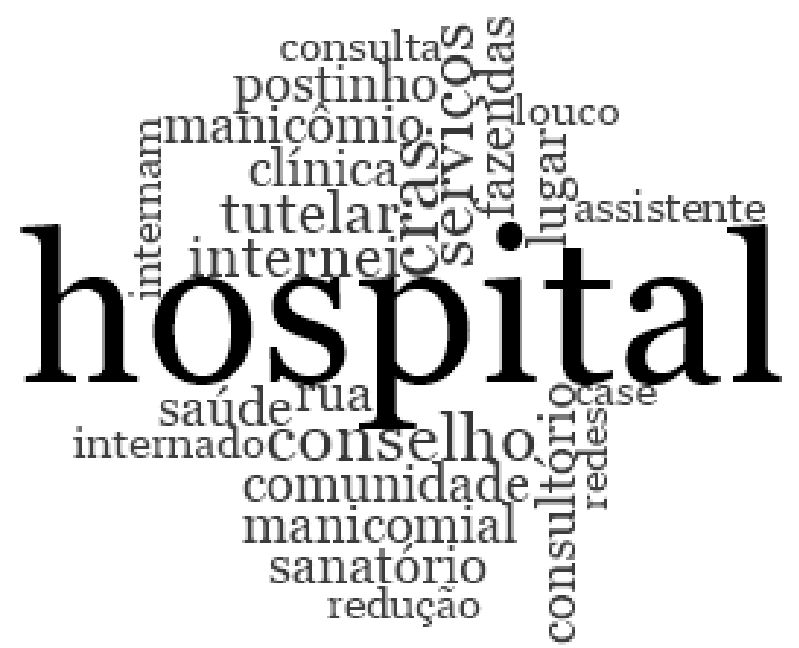

Figura 3 - Nuvem de palavras por frequência da codificação do sub-nó "Serviços utilizados adolescentes usuários de crack até chegar ao CAPS AD”, via QSR NVivo. 
13 | Ribeiro JP,Gomes GC, Eslabão AD, Oliveira NA

Os serviços de cunho manicomial mencionados são: hospital psiquiátrico, manicômio, fazendas e clínica.

Já passei em fazendas, já passei por um monte de lugar. Agora não me lembro, mas passei em bastante lugar. (A11)

Na clínica ali, no hospital psiquiátrico [...] fiquei lá 30 dias. Depois eu estive [no hospital geral], fiquei 15 dias lá, tomando remédio. (A14)

Eu internei ele no hospital psiquiátrico, fiz uma internação compulsória, e internei ele no hospital psiquiátrico e daí me passaram que assim que ele saísse do hospital ele deveria continuar o tratamento no CAPS. Aí eu retornei no CAPS para continuar o tratamento. $(\mathrm{F} 4)$

Fomos no sanatório e depois de lá saímos com o encaminhamento para o CAPS. (F6)

Geralmente eles chegam aqui pós hospital psiquiátrico, porque eles vão primeiro, direto para o hospital psiquiátrico. (P7)

Os serviços citados com menor frequência caracterizam-se como dispositivos públicos de proteção e atenção à saúde presentes na comunidade, tais como: postinho (Unidade Básica de Saúde - UBS), CRAS, conselho tutelar e consultório de rua.

$A$ assistente social ali do postinho que nos indicou. Olha, vocês tem que procurar o CAPS. (F8)

Primeiro eu fui no CRAS, acabava sempre indo no CRAS. Aí eu fui no CRAS e o CRAS me indicou para cá [CAPS AD]. (F2)

Eu fui no Conselho Tutelar e no Conselho eles me indicaram que eu tinha que levar ele para o CAPS. (F5)

Pela redução de danos e consultório de rua volta e meia chegam aqui, porque eles estão na boca, estão onde o adolescente mora. (P4)

\section{Discussão}

Autores $^{12}$ apontam que a demanda espontânea a partir do desejo e motivação do usuário é fundamental para o sucesso do tratamento no consumo abusivo de drogas, além disso, possuir o 
Trajetória dos adolescentes usuários de crack até o centro de atenção psicossocial álcool e drogas | 14

apoio da família para ser inserido no serviço é outro fator importante. Em uma revisão integrativa sobre redes sociais de usuários de drogas a família foi identificada como a única rede social de muitos usuários de drogas, após o rompimento de outros vínculos como, no trabalho, no relacionamento e com amigos.

Estudo realizado na Bahia com usuários de um CAPS AD, observou que a família é o suporte principal para a adesão ao tratamento, pois é vista como meio de apoio, afeto, direcionamento, alicerce para interação com o mundo, assim se tornando o meio para a reabilitação social. ${ }^{13}$

Por outro lado, o acesso dos adolescentes por meio da justiça é um ponto negativo, pois pode demonstrar uma busca tardia por tratamento, um desconhecimento dos serviços de saúde e/ou dificuldades de acesso e vínculo com os serviços de saúde. Pesquisa aponta um distanciamento entre os adolescentes e os serviços de cuidado em saúde mental, estas distâncias estão diretamente ligadas a escassez de serviços de saúde direcionados a essa população e a dificuldade de adesão, o modo pouco acolhedor dos profissionais, assim como a carência de formação dos mesmos, fragilidade na relação da dinâmica intersetorial entre saúde, trabalho, educação, esporte e justiça, além de intervenções/estratégias especificas para os jovens e adolescentes. $^{14}$

O presente estudo apontou que um dos motivos que leva os adolescentes ao tratamento no CAPS AD são os problemas comportamentais, os quais geram no seio familiar situações conflituosas com brigas e agressividades, ${ }^{15}$ a violência também foi evidenciada em outras pesquisas, ${ }^{16}$ além da questão do tráfico e o repudio do usuário de drogas pela sociedade. ${ }^{17}$ Tais achados evidenciam a complexidade do cuidado ao usuário de drogas e suas famílias, pois o sofrimento gerado pelo abuso de drogas é amplo, neste sentido, a violência, o preconceito e os conflitos familiares também se tornam objetos de intervenção da equipe de saúde. 
Outro motivo que leva o adolescente ao tratamento no CAPS AD está relacionado a busca por melhores condições de vida. Pesquisadores indicam que a busca para o tratamento ao uso prejudicial de drogas pode emergir de motivações externas como algum acontecimento e/ou a influência de alguma pessoa, tais como: conflitos no casamento e pedido de divórcio, dificuldades com os filhos, ou ainda pela ajuda de amigos e familiares que incentivaram o usuário a buscar tratamento. ${ }^{18}$

Se por um lado o tratamento pode vislumbrar ao adolescente uma possibilidade de melhores condições para buscar novos meios de reinserção social e cidadania, as constantes solicitações de internações compulsórias via judicial vão de encontro aos referenciais da reforma psiquiátrica e do cuidado em liberdade. Pesquisa documental realizada na Vara da Infância e Juventude de São Paulo para averiguar as decisões judiciais de processos para encaminhamento de crianças e adolescentes usuários problemáticos de drogas para tratamento nos serviços de saúde mental, constatou contradições entre as medidas socioeducativa de internação e o tratamento no CAPS. ${ }^{19}$

O CAPS como serviço de saúde mental em rede comunitária demanda a apropriação do território num sentido integrado, entretanto, o adolescente internado por uma medida socioeducativa não tem a possibilidade de se valer dos potenciais do território no processo de reabilitação psicossocial. Essa contradição restringe o tratamento de saúde mental à esfera meramente clínica e ambulatorial, realizada no interior do prédio do CAPS. Deste modo é preciso ampliar o olhar, pois o simples fato de um adolescente ser encaminhado ao CAPS não revela que o mesmo será cuidado de acordo com os referenciais da reforma psiquiátrica estando em medida socioeducativa. ${ }^{19}$

Além disso, o fato dos adolescentes utilizarem como porta de entrada na RAPS o hospital psiquiátrico e as fazendas terapêuticas traz à tona algumas questões emblemáticas nesta área como, a política de apaga incêndio que por meio de instituições excludentes retiram o usuário 
Trajetória dos adolescentes usuários de crack até o centro de atenção psicossocial álcool e drogas | 16

de drogas do seu meio social e das ruas e o pouco investimento na rede psicossocial de saúde mental e do cuidado no território por meio da prevenção e promoção da saúde.

O município desta pesquisa possui uma rede substitutiva em saúde mental com um número expressivo de serviços, mas o hospital psiquiátrico permanece na rede contrariando o que prevê a Lei 10.2016 de 2001 sobre o fechamento do manicômio mediando a criação de serviços substitutivos. Neste sentido, alguns questionamentos são relevantes como: por que a estrutura do hospital psiquiátrico permanece no município? Os serviços substitutivos estão sendo resolutivos?

Deste modo, a permanência do hospital psiquiátrico na rede leva muitas pessoas a utilizálo por questões culturais e histórico acerca desse serviço, além disso, pode revelar um desconhecimento pelas famílias sobre a rede de serviços substitutivos em saúde mental, bem como a falta de vínculo a estes dispositivos.

Essas contradições presentes no cotidiano de trabalho, na complexidade do campo da saúde mental infanto-juvenil, é fruto de heranças históricas na operacionalização do modelo de atenção a usuários de álcool e drogas. $\mathrm{Na}$ composição desse modelo podem-se ver instituições de caráter fechado, que cresceram historicamente focadas na criminalização e repressão de usuários de álcool e drogas, e também, instituições cujo cuidado é operado em rede de saúde mental constituída por equipamentos abertos (CAPS AD) que trabalham na lógica da redução de danos, do modelo de atenção psicossocial. ${ }^{20}$

Os resultados desta pesquisa evidenciam que o modelo psicossocial e modelo asilar andam juntos sendo preciso maior investimento no cuidado no território, nas práticas de prevenção e promoção a saúde. A redução de danos como estratégia de cuidado e norteadora da Política Nacional de Saúde Mental Álcool e outras Drogas pode ser a inovação para o cuidado, prevenção e promoção da saúde nos territórios com grande vulnerabilidade social e tráfico de drogas. 
Mas, para tanto, é preciso investir mais em equipes de redução de danos, em cuidados no território e em formação permanente dos profissionais que estão inseridos nestas atividades, de forma a desenvolver as habilidades de escuta, comunicação e de análises profundas, evitando o risco de realizar velhas práticas em novos modelos, que estão mais relacionados à linha da penalização da vida, segregação e da psiquiatrização. ${ }^{21}$

\section{Considerações finais}

A análise da trajetória percorrida pelos adolescentes usuários de crack até o tratamento no CAPS AD apontou a relevância de conhecer a forma como eles chegam ao serviço; os motivos que os levaram para tratamento e os serviços utilizados até chegar ao mesmo.

A forma como o adolescente usuário de crack chega ao CAPS AD evidenciou que a RAPS necessariamente exige uma articulação intersetorial, pois os jovens acessam o serviço não só de forma espontânea, mas principalmente devido a encaminhamentos realizados por órgãos de proteção e justiça e da rede socioassistencial. Destacam-se entre os motivos que levam o adolescente usuário de crack para tratamento o desejo do próprio adolescente em investir num projeto de vida melhor, pedidos da justiça e problemas comportamentais.

Por esta razão, faz-se imperativo que o CAPS AD articule ações de prevenção ao uso de substâncias e promoção da saúde junto a serviços existentes no território (Escolas, Unidades Básicas de Saúde, Estratégia Saúde da Família, Centro de Referência de Assistência Social, entre outros.), explorando e valorizando os projetos de vida dos adolescentes de forma que o mesmo possa vislumbrar possibilidades de concretizá-los e (re)inserir-se socialmente.

Os serviços utilizados pelos adolescentes usuários de crack até chegar ao CAPS AD revelam uma cultura de tratamento manicomial, em que o hospital psiquiátrico constitui-se na principal forma de entrada no serviço. Não raro, associadas as solicitações de internações 
Trajetória dos adolescentes usuários de crack até o centro de atenção psicossocial álcool e drogas | 18

compulsórias via judicial; o que vai de encontro aos referenciais da reforma psiquiátrica e do cuidado em liberdade.

Para superar práticas excludentes e retrógradas de cuidado, mostra-se importante o investimento nos serviços e ações desenvolvidas pela RAPS, dando-lhes maior visibilidade e acesso aos jovens; como também, capacitar seus profissionais com habilidades e competências para buscar novos meios de (re)inserção social e cidadania aos adolescentes.

O presente estudo possui como limitação o fato de retratar uma realidade local e, embora os dados apresentados possam refletir fatos existentes em outras localidades, não pode ser generalizado devido as especificidades do município estudado.

\section{Referências}

1. Costa-Rosa A. Atenção psicossocial além da reforma psiquiátrica: contribuições a uma clínica crítica dos processos de subjetivação na saúde coletiva [Internet]. São Paulo: Unesp; 2013 [acesso em 2018 fev 08]. 334 p. Disponível em: http://medicalizacao.org.br/wp-content/uploads/2014/08/0.-Costa-Rosa-A.Aten\%C3\%A7\%C3\%A3o-Psicossocial-al\%C3\%A9m-da-Reforma-Psiqui\%C3\%A1trica-vers\%C3\%A3orevisada.pdf

2. Ministério da Saúde (BR). Conheça a RAPS: Rede de Atenção Psicossocial [Internet]. Brasília (DF): Ministério da Educação; 2013 [acesso 2017 nov 10]. Disponível em: http://bvsms.saude.gov.br/bvs/folder/conheca_raps_rede_atencao_psicossocial.pdf

3. Ministério da Saúde (BR). Guia estratégico para o cuidado de pessoas com necessidades relacionadas ao consumo de álcool e outras drogas: guia AD [Internet]. Brasília (DF): Ministério da Saúde; 2015 [acesso 2017 nov 10]. Disponível em: http://bvsms.saude.gov.br/bvs/publicacoes/guia_estrategico_cuidado_pessoas_necessidades.pdf

4. Bernardi AB, Kanan LA. Características dos serviços públicos de saúde mental (Capsi, Capsad, Caps III) do estado de Santa Catarina. Saúde Debate [Internet]. 2015 [acesso 2018 fev 08];39(107):1105-16. Disponível em:

http://www.scielo.br/scielo.php?script=sci_arttext\&pid=S0103-11042015000401105

5. Paim BR, Porta DD, Sarzi DM, Cardinal MF, Siqueira DF, Mello AL, et al. Atendimento ao adolescente usuário de substancias psicoativas: papel do centro de atenção psicossocial. Cogitare Enferm 
19 | Ribeiro JP,Gomes GC, Eslabão AD, Oliveira NA

[Internet]. 2017 [acesso 2019 abril 07];(22)1:e48011. Disponível em: http://revistas.ufpr.br/cogitare/article/view/48011

6. Delany-Moretlwe S, Cowan FM, Busza J, Bolton-Moore C, Kelley K, Fairlie L. Providing comprehensive health services for young key populations: needs, barriers and gaps. J Int AIDS Soc [Internet]. 2015 [acesso 2018 jan 03];18(2 Suppl 1):19833. Disponível em: https://www.ncbi.nlm.nih.gov/pmc/articles/PMC4344539/pdf/JIAS-18-19833.pdf

7. Bard ND, Antunes B, Roos CM, Olschowsky A, Pinho LB. Estigma e preconceito: vivência dos usuários de crack. Rev Latinoam Enferm [Internet]. 2016 [acesso em 2019 abril 07];24(e2680):2-7. Disponível em: http://www.scielo.br/pdf/rlae/v24/pt_0104-1169-rlae-0852-2680.pdf

8. Flick W. Qualidade na pesquisa qualitativa. Porto Alegre: Artmed; 2009.

9. Guizzo BS, Krziminski CO, Oliveira DLLC. O software QSR NVIVO 2.0 na análise qualitativa de dados: ferramenta para a pesquisa em ciências humanas e da saúde. Rev Gaúch Enferm [Internet]. 2003 [acesso 2017 jun 26];24(1):53-60. Disponível em: http://seer.ufrgs.br/RevistaGauchadeEnfermagem/article/view/4437

10. Minayo MCS. O desafio do conhecimento: pesquisa qualitativa em saúde. $13^{\text {a }}$ ed. São Paulo (SP): Hucitec; 2013.

11. Brasil. Ministério da Saúde. Conselho Nacional de Saúde. Resolução CNS n. 466, de 12 de dezembro de 2012. Aprova as seguintes diretrizes e normas técnicas regulamentadoras de pesquisas envolvendo seres humanos. Brasília (DF): Ministério da Saúde; 2012.

12. Loures BP, Costa PHA, Ronzani TM. As redes sociais no cuidado aos usuários de drogas: revisão sistemática. Psicol Estud [Internet]. 2016 [acesso 2018 fev 08];21(1):29-39. Disponível em: http://www.redalyc.org/pdf/2871/287146384006.pdf

13. Lima JL, Carvalho PAL, Santos VTC, Oliveira MAF, Malhado SCB, Santana EM, et al. Perception of drug users on family: a phenomenological study. Rev Bras Enferm [Internet]. 2018 [acesso em $2019 \mathrm{abr}$ 07];71(5):2094-100. Disponível em: http://www.scielo.br/pdf/reben/v71s5/0034-7167-reben-71-s5-2094.pdf

14. Paiano M, Maftum MA, Haddad MCL, Marcon SS. Ambulatório de saúde mental: fragilidades apontadas por profissionais. Texto \& Contexto Enferm [Internet]. 2016 [acesso em 2019 abril 07]; 25(3):210. Disponível em: http://www.scielo.br/pdf/tce/v25n3/pt_0104-0707-tce-25-03-0040014.pdf

15. Golub A, Dunlap E, Benoit E. Drug use and conflict in inner-city African-American relationships in the 2000. J Psychoactive Drugs [Internet]. 2010 [acesso 2018 fev 02];42(3):327-37. Disponível em: https://www.ncbi.nlm.nih.gov/pmc/articles/PMC3743426/pdf/nihms284812.pdf 
Trajetória dos adolescentes usuários de crack até o centro de atenção psicossocial álcool e drogas | 20

16. Predebon J, Giongo C. A família com filhos adolescentes em conflito com a lei: contribuições de pesquisas brasileiras. Pensando Fam [Internet]. 2015 [acesso 2018 fev 02];19(1):88-104. Disponível em: http://pepsic.bvsalud.org/pdf/penf/v19n1/v19n1a08.pdf

17. Souza WC, Alencar IM, Ribeiro PE. Cuidado integral à saúde de usuários de crack e outras drogas: ação de rede entre consultório de rua e atenção básica.Contrib Cienc Soc [Internet]. 2015 nov [acesso 2018 fev 08];1-18. Disponível em: http://www.eumed.net/rev/cccss/2015/04/crack.html

18. Eckhardt NS, Raupp L. Itinerários terapêuticos de usuários de drogas na região Sul do Brasil. Psicol Saúde Doenças [Internet]. 2017 [acesso 2018 fev 08];18(1):263-77. Disponível em: http://www.scielo.mec.pt/pdf/psd/v18n1/v18n1a21.pdf

19. Soares RH, Oliveira MAF, Leite KC, Nascimento GCG. Medidas judiciais atinentes à atenção em saúde mental de adolescentes em conflito com a lei. Interface (Botucatu) [Internet]. 2017 [acesso 2019 abril 07];21(60):123-31. Disponível em: http://www.scielo.br/pdf/icse/v21n60/1807-5762-icse-1807576220150636.pdf

20. Bastos IT, Sarubbi VJ, Oliveira TGP, Delfini PSS, Muylaert CJ, Reis AOA. Identidade do cuidado em Centro de Atenção Psicossocial Infantojuvenil para usuários de álcool e drogas. Rev Esc Enferm USP [Internet]. 2014 [acesso 2018 fev 02];48(2):121-7. Disponível em: http://www.scielo.br/pdf/reeusp/v48nspe2/pt_0080-6234-reeusp-48-nspe2-00116.pdf

21. Veronese JRP. O adolescente autor de ato infracional sob a perspectiva da intersetorialidade. Rev Direito Unisc [Internet]. 2015 [acesso 2018 fev 02];3(47):125-43. Disponível em: https://online.unisc.br/seer/index.php/direito/article/view/6430/4399

\section{Autor correspondente}

Juliane Portella Ribeiro

E-mail: ju_ribeiro1985@hotmail.com

Endereço: Gomes Carneiro, nํㅜㄹ, 2 andar. Universidade Federal de Pelotas, Faculdade de

Enfermagem - Centro, Pelotas - RS.

CEP: $96010-610$

\section{Contribuições de Autoria}

1 - Juliane Portella Ribeiro -

Contribuiu na concepção, projeto, análise, interpretação dos dados e aprovação final da versão a ser publicada.

2 - Giovana Calcagno Gomes -

Contribuiu na concepção, projeto, análise, interpretação dos dados e aprovação final da versão a ser publicada. 
21 | Ribeiro JP,Gomes GC, Eslabão AD, Oliveira NA

3 - Adriane Domingues Eslabão -

Contribuiu na redação do artigo e revisão crítica relevante do conteúdo intelectual, bem como da aprovação final da versão a ser publicada.

4 - Naiana Alves Oliveira -

Contribuiu na redação do artigo e revisão crítica relevante do conteúdo intelectual, bem como da aprovação final da versão a ser publicada.

\section{Como citar este artigo}

Ribeiro JP,Gomes GC, Eslabão AD, Oliveira NA. Trajetória dos adolescentes usuários de crack até o centro de atenção psicossocial álcool e drogas. Rev. Enferm. UFSM. 2019 [Acesso em: 2019 jun 15]; vol ex:p1-p21. DOI:https://doi.org/10.5902/2179769232526 